\title{
ON THE STABILITY OF THE LINEAR MAPPING IN BANACH SPACES
}

\author{
THEMISTOCLES M. RASSIAS ${ }^{1}$
}

\begin{abstract}
Let $E_{1}, E_{2}$ be two Banach spaces, and let $f: E_{1} \rightarrow E_{2}$ be a mapping, that is "approximately linear". S. M. Ulam posed the problem: "Give conditions in order for a linear mapping near an approximately linear mapping to exist". The purpose of this paper is to give an answer to Ulam's problem.
\end{abstract}

THEOREM. Consider $E_{1}, E_{2}$ to be two Banach spaces, and let $f: E_{1} \rightarrow E_{2}$ be a mapping such that $f(t x)$ is continuous in $t$ for each fixed $x$. Assume that there exists $\theta \geqslant 0$ and $p \in[0,1)$ such that

$$
\frac{\|f(x+y)-f(x)-f(y)\|}{\|x\|^{p}+\|y\|^{p}} \leqslant \theta, \quad \text { for any } x, y \in E_{1} .
$$

Then there exists a unique linear mapping $T: E_{1} \rightarrow E_{2}$ such that

$$
\frac{\|f(x)-T(x)\|}{\|x\|^{p}} \leqslant \frac{2 \theta}{2-2^{p}}, \text { for any } x \in E_{1} .
$$

Proof. Claim that

$$
\frac{\left\|\left[f\left(2^{n} x\right)\right] / 2^{n}-f(x)\right\|}{\|x\|^{p}} \leqslant \theta \sum_{m=0}^{n-1} 2^{m(p-1)}
$$

for any integer $n$, and some $\theta \geqslant 0$. The verification of (3) follows by induction on $n$. Indeed the case $n=1$ is clear because by the hypothesis we can find $\theta$, that is greater or equal to zero, and $p$ such that $0 \leqslant p<1$ with

$$
\frac{\|[f(2 x)] / 2-f(x)\|}{\|x\|^{p}} \leqslant \theta .
$$

Assume now that (3) holds and we want to prove it for the case $(n+1)$. However this is true because by (3) we obtain

$$
\frac{\left\|\left[f\left(2^{n} \cdot 2 x\right)\right] / 2^{n}-f(2 x)\right\|}{\|2 x\|^{p}} \leqslant \theta \sum_{m=0}^{n-1} 2^{m(p-1)},
$$

therefore

Received by the editors December 1, 1977.

AMS (MOS) subject classifications (1970). Primary 47H15; Secondary 39A15.

Key words and phrases. Approximately linear mapping.

${ }^{1}$ Research supported by the University of California at Berkeley while the author was a postdoctoral Research Fellow at the Department of Mathematics of the University of California at Berkeley.

๑ American Mathematical Society 1978 


$$
\frac{\left\|\left[f\left(2^{n+1} x\right)\right] / 2^{n+1}-\frac{1}{2} f(2 x)\right\|}{\|x\|^{p}} \leqslant \theta \sum_{m=1}^{n} 2^{m(p-1)} .
$$

By the triangle inequality we obtain

$$
\begin{aligned}
\left\|\frac{1}{2^{n+1}}\left[f\left(2^{n+1} x\right)\right]-f(x)\right\| \leqslant & \left\|\frac{1}{2^{n+1}}\left[f\left(2^{n+1} x\right)\right]-\frac{1}{2}[f(2 x)]\right\| \\
& +\left\|\frac{1}{2}[f(2 x)]-f(x)\right\| \leqslant \theta\|x\|^{p} \sum_{m=0}^{n} 2^{m(p-1)} .
\end{aligned}
$$

Thus

$$
\frac{\left\|\left[f\left(2^{n+1} x\right)\right] / 2^{n+1}-f(x)\right\|}{\|x\|^{p}} \leqslant \theta \sum_{m=0}^{n} 2^{m(p-1)}
$$

and (3) is valid for any integer $n$. It follows then that

$$
\frac{\left\|\left[f\left(2^{n} x\right)\right] / 2^{n}-f(x)\right\|}{\|x\|^{p}} \leqslant \frac{2 \theta}{2-2^{p}},
$$

because $\sum_{m=0}^{\infty} 2^{m(p-1)}$ converges to $2 /\left(2-2^{p}\right)$, as $0 \leqslant p<1$. However, for $m>n>0$,

$$
\begin{aligned}
\left\|\frac{1}{2^{m}}\left[f\left(2^{m} x\right)\right]-\frac{1}{2^{n}}\left[f\left(2^{n} x\right)\right]\right\| & =\frac{1}{2^{n}}\left\|\frac{1}{2^{m-n}}\left[f\left(2^{m} x\right)\right]-\left[f\left(2^{n} x\right)\right]\right\| \\
& <2^{n(p-1)} \cdot \frac{2 \theta}{2-2^{p}}\|x\|^{p}
\end{aligned}
$$

Therefore

$$
\lim _{n \rightarrow \infty}\left\|\frac{1}{2^{m}}\left[f\left(2^{m} x\right)\right]-\frac{1}{2^{n}}\left[f\left(2^{n} x\right)\right]\right\|=0 .
$$

But $E_{2}$, as a Banach space, is complete, thus the sequence $\left\{\left[f\left(2^{n} x\right)\right] / 2^{n}\right\}$ converges. Set

$$
T(x) \equiv \lim _{n \rightarrow \infty} \frac{1}{2^{n}}\left[f\left(2^{n} x\right)\right]
$$

It follows that

$$
\begin{aligned}
\left\|f\left[2^{n}(x+y)\right]-f\left[2^{n} x\right]-f\left[2^{n} y\right]\right\| & \leqslant \theta\left(\left\|2^{n} x\right\|^{p}+\left\|2^{n} y\right\|^{p}\right) \\
& =2^{n p} \theta\left(\|x\|^{p}+\|y\|^{p}\right) .
\end{aligned}
$$

Therefore

$$
\frac{1}{2^{n}}\left\|f\left[2^{n}(x+y)\right]-f\left[2^{n} x\right]-f\left[2^{n} y\right]\right\| \leqslant 2^{n(p-1)} \cdot \theta\left(\|x\|^{p}+\|y\|^{p}\right)
$$

or

$\lim _{n \rightarrow \infty} \frac{1}{2^{n}}\left\|f\left[2^{n}(x+y)\right]-f\left[2^{n} x\right]-f\left[2^{n} y\right]\right\| \leqslant \lim _{n \rightarrow \infty} 2^{n(p-1)} \theta\left(\|x\|^{p}+\|y\|^{p}\right)$

or 


$$
\left\|\lim _{n \rightarrow \infty} \frac{1}{2^{n}} f\left[2^{n}(x+y)\right]-\lim _{n \rightarrow \infty} \frac{1}{2^{n}} f\left[2^{n} x\right]-\lim _{n \rightarrow \infty} \frac{1}{2^{n}} f\left[2^{n} y\right]\right\|=0
$$

or

$$
\|T(x+y)-T(x)-T(y)\|=0 \text { for any } x, y \in E_{1}
$$

or

$$
T(x+y)=T(x)+T(y) \text { for all } x, y \in E_{1} .
$$

Since $T(x+y)=T(x)+T(y)$ for any $x, y \in E_{1}, T(r x)=r T(x)$ for any rational number $r$. Fix $x_{0} \in E_{1}$ and $\rho \in E_{2}^{*}$ (the dual space of $E_{2}$ ). Consider the mapping

$$
\mathbf{R} \ni t \mapsto \rho(T(t x))=\phi(t) .
$$

Then $\phi: \mathbf{R} \rightarrow \mathbf{R}$ satisfies the property that $\phi(a+b)=\phi(a)+\phi(b)$, i.e. $\phi$ is a group homomorphism. Moreover $\phi$ is a Borel function, because of the following reasoning. Let $\phi(t)=\lim _{n \rightarrow \infty} \rho\left(f\left(2^{n} t x_{0}\right)\right) / 2^{n}$ and set $\phi_{n}(t)=$ $\rho\left(f\left(2^{n} t x_{0}\right)\right) / 2^{n}$. Then $\phi_{n}(t)$ are continuous functions. But $\phi(t)$ is the pointwise limit of continuous functions, thus $\phi(t)$ is a Borel function. It is a known fact that if $\phi: \mathbf{R}^{n} \rightarrow \mathbf{R}^{n}$ is a function such that $\phi$ is a group homomorphism, i.e. $\phi(x+y)=\phi(x)+\phi(y)$ and $\phi$ is a measurable function, then $\phi$ is continuous. In fact this statement is also true if we replace $\mathbf{R}^{n}$ by any separable, locally compact abelian group (see for example: W. Rudin [3]). Therefore $\phi(t)$ is a continuous function. Let $a \in \mathbf{R}$. Then $a=\lim _{n \rightarrow \infty} r_{n}$, where $\left\{r_{n}\right\}$ is a sequence of rational numbers. Hence

$$
\phi(a t)=\phi\left(t \lim _{n \rightarrow \infty} r_{n}\right)=\lim _{n \rightarrow \infty} \phi\left(t r_{n}\right)=\left(\lim _{n \rightarrow \infty} r_{n}\right) \phi(t)=a \phi(t) .
$$

Therefore $\phi(a t)=a \phi(t)$ for any $a \in \mathbf{R}$. Thus $T(a x)=a T(x)$ for any $a \in \mathbf{R}$. Hence $T$ is a linear mapping.

From (5) we obtain

$$
\lim _{n \rightarrow \infty} \frac{\left\|\left[f\left(2^{n} x\right)\right] / 2^{n}-f(x)\right\|}{\|x\|^{p}} \leqslant \lim _{n \rightarrow \infty} \frac{2 \theta}{2-2^{p}}
$$

or equivalently,

$$
\frac{\|T(x)-f(x)\|}{\|x\|^{p}} \leqslant \varepsilon, \quad \text { where } \varepsilon=\frac{2 \theta}{2-2^{p}},
$$

Thus we have obtained (2).

We want now to prove that $T$ is the unique such linear mapping. Assume that there exists another one, denoted by $g: E_{1} \rightarrow E_{2}$ such that $T(x) \not g(x)$, $x \in E_{1}$. Then there exists a constant $\varepsilon_{1}$, greater or equal to zero, and $q$ such that $0 \leqslant q<1$ with

$$
\frac{\|g(x)-f(x)\|}{\|x\|^{q}} \leqslant \varepsilon_{1} .
$$

By the triangle inequality and (6) we obtain 


$$
\|T(x)-g(x)\| \leqslant\|T(x)-f(x)\|+\|f(x)-g(x)\| \leqslant \varepsilon\|x\|^{p}+\varepsilon_{1}\|x\|^{q} .
$$

Therefore

$$
\begin{aligned}
\|T(x)-g(x)\| & =\left\|\frac{1}{n}[T(n x)]-\frac{1}{n}[g(n x)]\right\|=\frac{1}{n}\|T(n x)-g(n x)\| \\
& \leqslant \frac{1}{n}\left(\varepsilon\|n x\|^{p}+\varepsilon_{1}\|n x\|^{q}\right)=n^{p-1} \varepsilon\|x\|^{p}+n^{q-1} \varepsilon_{1}\|x\|^{q} .
\end{aligned}
$$

Thus $\lim _{n \rightarrow \infty}\|T(x)-g(x)\|=0$ for all $x \in E_{1}$ and hence $T(x) \equiv g(x)$ for all $x \in E_{1}$. Q.E.D.

This solves a problem posed by S. M. Ulam [4], [5]: When does a linear mapping near an "approximately linear" mapping exist? The case $p=0$ was answered by D. H. Hyers [1]. Thus we have succeeded here to give a generalized solution to Ulam's problem.

ACKNOWLEDGMENT. It is my pleasure to express my thanks to Professor D. H. Hyers, who read a previous version of the manuscript, for his helpful comments.

\section{REFERENCES}

1. D. H. Hyers, On the stability of the linear functional equation, Proc. Nat. Acad. Sci. U.S.A. 27 (1941), 222-224.

2. T. M. Rassias, Vector fields on Banach manifolds (to appear).

3. W. Rudin, Fourier analysis on groups, Interscience, New York, 1962.

4. S. M. Ulam, Problems in modern mathematics, Chapter VI, Science Editions, Wiley, New York, 1960.

5. __ Sets, numbers, and universes. Selected works, Part III, The MIT Press, Cambridge, Mass. and London, 1974.

6. K. Yosida, Functional analysis, Springer, Berlin-Göttingen-Heidelberg, 1965.

Department of Mathematics, University of California at Berkeley, Berkeley, CaliFORNIA 94720

Current address: Pellana, Sparta, Lacony, Greece 